\title{
OBSERVATION AND CURES OF WAKEFIELD EFFECTS IN THE SLC LINAC*
}

\author{
John T. Seeman
}

Stanford Linear Accelerator Center, Stanford University, Stanford, California, 94309

\begin{abstract}
The acceleration and transport of beams with high currents and low emittances are very carefully controlled for the SLC Linac ${ }^{1}$. Both longitudinal and transverse wakefields affect strongly the trajectory and emittance corrections used for operations during collisions. Longitudinal beam loading introduces an internal energy spread. This loading produces the expected doubled peaked energy spectra while reducing the overall available energy for acceleration. Transverse wakefields cause rapid oscillation amplitude growth with injection errors but can be ameliorated with transverse wakefield (BNS) damping. Transverse wakefields cause an apparent change in the lattice strength, affecting the interpretation of lattice diagnostics. Oscillations also produce non-gaussian transverse density distributions, which must be controlled for emittance and background reduction. Finally, the small misalignments of the accelerating structure enlarge the emittances of the beam. They can be controlled through off-axis trajectories and through systematic waveguide movements.
\end{abstract}

\subsection{Acceleration}

The energy $E$ of particles accelerated in a linac after $n$ klystrons is given by ${ }^{2}$

$$
E(z)=E_{i n j}+\sum_{i=1}^{n}\left[\Delta E_{i} \cos \left(\phi_{i}+\phi(z)\right)+\Delta s_{i} \int_{z}^{\infty} W_{l}\left(z^{\prime}-z\right) \rho\left(z^{\prime}\right) d z^{\prime}\right]
$$

where $E_{i n j}$ is the injection energy, $\phi_{i}$ are the klystron phases-which are free parameters-, $\phi(z)=$ $2 \pi z / \lambda_{R F}$, where $\lambda_{R F}$ is the RF wavelength, $z$ is the longitudinal position along the bunch, $\Delta E_{i}$ is the maximum energy gain in the distance $\Delta \mathrm{s}_{\mathrm{i}}$ (one klystron), $\rho(\mathrm{z})$ is the longitudinal density distribution, and the last term on the right-hand side is the longitudinal wakefield contribution to the particle energy. The longitudinal wakefield contribution $\mathrm{W}_{1}$ arises from all particles in the bunch precedirig the one of interest. The longitudinal wakefield depends on the shapes of the RF cavities and surrounding vacuum hardware ${ }^{3}$. For the SLC $\Delta \mathrm{E}_{\mathrm{i}}=20 \mathrm{MeV} / \mathrm{m}, \lambda_{\mathrm{RF}}=10 \mathrm{~cm}$, and $\Delta \mathrm{s}_{\mathrm{i}}=12 \mathrm{~m} . \rho(\mathrm{z})$ is a gaussian at low currents with $\sigma_{\mathrm{z}}=1 \mathrm{~mm}$. At $5 \times 10^{10} \mathrm{e}^{-}$the longitudinal wakefields decelerates the tail by $2 \mathrm{GeV}$ over the $3 \mathrm{~km}$ linic. The $230 \mathrm{klystrons}$ can accelerate low currents to $56 \mathrm{GeV}$.

When the SLC linac accelerates three bunches, the longitudinal loading affects the energy differences between the bunches ${ }^{1}$. Trailing bunches see a lower accelerating field. The energy extracted from the RF cavities by the bean must be replenished by an RF power source but there is a finite filling tirne. The method to equalize the bunch energies is to increase the klystron pulse amplitude with time. In the SLC the pulsed SLED system ${ }^{5}$ provides a time varying field. This is used to make the two colliding bunches in the SLC have equal energies ${ }^{4}$.

\subsection{Energy spectrum}

At high currents the longitudinal wakefield decelerates the longitudinal tail of the bunch. Thus, in order to minimize the energy spread, the mean phase of the bunch is moved forward of the RF crest. The energy distribution in the bunch develops a complicated shape due to the nonlinear wakefield, particle density distribution, and RF sine wave. These distributions have been calculated

* Work supported by the Department of Energy, contract DE-AC03-76SF00515.

Contributed talk at the 5th ICFA Advanced Beam Dynamics Workshop on "Effects of Errors in Accelerators, Their Diagnosis and Correction.", Corpus Christi, TX, October 3-8, 1991. 
for and observed in the SLC linac for low and high currents. An example ${ }^{4}$ of a beam with a "double homed" energy distribution at $3 \times 10^{10} \mathrm{e}^{-}$and $\sigma_{\mathrm{z}}=1 \mathrm{~mm}$ is shown in Figure 1. The changes in the optimum linac phase for the minimum energy spread as a function of current can be up to 10 to 15 degrees. This shift permits striy phase jitter in the klystrons to produce energy jitter in a linac. At high currents, the longitudinal bunch distributions are not true gaussians and careful integrals are required to extracted the energy spectra 6 .

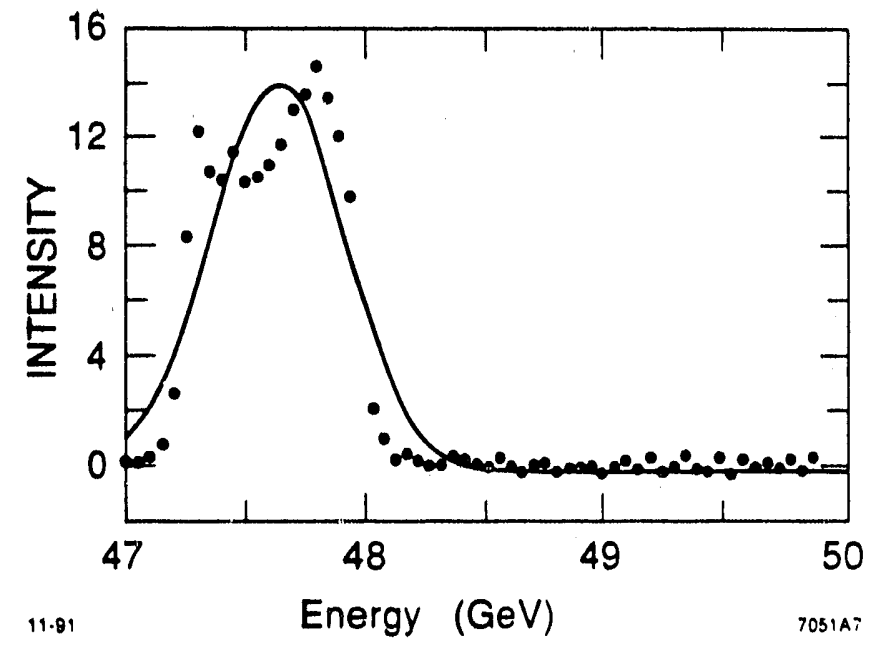

Figure 1 At high charge the measured energy spectrum in the SLC has the characteristic double peaked structure as determined by non-linear longitudinal wakefields.

\subsection{Transverse Oscillations}

The transverse equation of motion for particles under the influence of transverse wakefields is

$$
\frac{d^{2}}{d s^{2}} x(z, s)+k^{2}(z, s) x(z, s)=\frac{r_{e}}{\gamma(z, s)} \int_{z}^{\infty} d z^{\prime} \rho\left(z^{\prime}\right) W_{r}\left(z^{\prime}-z\right)\left(x\left(z^{\prime}, s\right)-d(s)\right),
$$

where $\mathrm{x}$ is the transverse particle displacement which depends on where in the bunch the particle is located and where along the accelerator the bunch is located, $k$ is the lattice strength, $\rho$ is the longitudinal particle density, $\gamma=\mathrm{E} / \mathrm{mc}^{2}, \mathrm{r}_{\mathrm{e}}$ is the classical electron radius, $s$ is the distance along the accelerator, and $\mathrm{W}_{\mathrm{r}}$ is the transverse wakefield which depends on the separation of the leading and trailing particles. $W_{r}$ grows nearly linearly for beams with millimeter bunch lengths for the SLAC structure ${ }^{3} \cdot d(z)$ is the misalignment of the accelerator along the linac. The acceleration is approximately linear, $\gamma(s)=\gamma(0)+G s$, where $G$ is the accelerating gradient. In Equation (2) the distance to double the energy is assumed to be long compared to the betatron wavelength, or $k \gg G$.

The driving force on the right hand side is proportional to the transverse offset of all the leading particles in the RF structure of the accelerator. Thus, there is the potential for resonant excitation of the back of the bunch by the head. There are many mechanisms to start an oscillation. The energy spread in the bunch affects the equation of motion. Also, the motion will develop differently if the initial deflection is made at different locations in the accelerator.

A complete solution to Equation (4) requires a computer given the non-gaussian longitudinal distribution, wakefields, and acceleration. Approximate analytical solutions can be obtained by dividing the beam into several longitudinal slices. Here, a model with three longitudinal slices without acceleration is reviewed. If the bunch has a total charge of $N$, then the center ' $c$ ' slice is assigned $N / 2$ charges and the head ' $h$ ' and tail ' $t$ ' slices have $N / 4$ charges each. They are all spaced apart by $\sigma_{z}$. The three solutions to the equations of motion assuming a linear transverse wakefield and an initial deflection $\mathrm{k} \mathrm{x}_{0}$ are 
$x_{h}=x_{0} \sin (k s) ; x_{c}=x_{0}\left[A_{c} \sin (k s)-B_{c} s \cos (k s)\right] ; x_{t}=x_{0}\left[A_{t} \sin (k s)-B_{t} s \cos (k s)-C_{t} s^{2} \sin (k s)\right]$

where the coefficients $A_{c}, A_{t}, B_{c}$, and $B_{t}$ are linearly proportional to the bunch charge and the transverse wakefield given by $D$. The coefficient $C_{t}$ is quadratic in $D$.

$$
\mathrm{D}=\mathrm{e} \frac{\mathrm{N} \mathrm{W}_{\mathrm{I}} \sigma_{\mathrm{z}}}{4 \mathrm{E}_{0}}
$$

There are three important conclusions: 1) The variable $x_{0}$ factors from all the solutions, which means that the solutions are linear in the initial amplitude. 2) Each additional slice adds one higher power of $D$ ( or $\mathrm{W}_{\mathrm{r}} \mathrm{N}$ ) and $\mathrm{s}$, indicating the nonlinearity in bunch charge and distance along the accelerator. 3) The core lags 90 degrees behind the head and the tail, ultimately, lags 180 degrees behind the head. Conclusion 2 suggests an exponential growth of the tail of the beam in the limit of many slices. This resonant blowup at high currents was been observed in the SLC, as shown in Figure 2. Simulations and observations are in good agreement supporting an approximate exponential trajectory increase with distance ${ }^{7}$. In this condition the SLC is very susceptible to injection jitter.

\subsection{Transverse wakefield (BNS) damping}

A technique to control this resonant growth is called BNS damping (named after the inventors: V. Balakin, A. Novokhatsky, and V. Smirnov) ${ }^{8}$. BNS damping lowers the energy of the back of the bunch by accelerating the bunch behind the crest of the RF early in the linac and then ahead of the RF crest downstream to restore the energy spread at the end. With the back of the bunch low in energy, the wakefield forces in Equation (2), which act to defocus particles, are counterbalanced by the increased quadrupole focusing of the back of the bunch because of its low energy. The resonant excitation is then reduced. It is not possible to perfectly cancel the forces over the whole bunch so some enlargement is expected.

The solutions to the three particle model with BNS damping added can be determined. BNS damping enters the equations in the lattice constants $k$. The energies of the three particles are different and so are their lattice constants. The lattice constant for the head is set to $k$, for the core $k-\Delta k_{c}$, and for the tail $k-\Delta k_{t}$. The solutions to the three equations change from Equation (3). The head just oscillates at its frequency, $k$. The core oscillates as the sum of two sine waves with frequencies $k-\Delta k_{c}$ and $k$. The longitudinal tail oscillates as the sum of three sine waves of frequencies $k, k-\Delta k_{c}$, and $k-\Delta k_{t}$. These oscillations are bounded and beat along the accelerator. With special conditions, all particles oscillate in phase with the same amplitude. To make the head and core particles oscillate together, $\Delta \mathrm{k}_{\mathrm{c}}$ is adjusted to make identical trajectories. $\Delta \mathrm{k}_{\mathrm{c}}=\mathrm{e} \mathrm{N} \mathrm{W}_{\mathrm{r}} \sigma_{\mathrm{z}} /\left(4 \mathrm{E}_{0} \mathrm{k}\right)$ [i.e. BNS condition]. The core wants to have an energy that makes the lattice appear stronger, i.e. a lower energy. To make the third particle oscillate like the first two then $\Delta k_{t}=4 \Delta k_{c}$. The factor of four comes from twice the wakefield effect of the head on the tail and twice the effect of the core on the tail because of the increased charge. These solutions indicate that the energy differences over the length of the bunch need careful tailoring. This is the essence of the notion of autophasing.

The strong effect of this transverse damping is shown in Figure 2 where beams with and without BNS damping are made to oscillate in the SLC ${ }^{9}$. BNS damping works very well and is used continuously in the SLC. With full BNS damping conditions, oscillations of a bunch have been studied in detail. Four measured oscillations, with identical initial conditions, are shown in Figure 3 for four different bunch currents. The BNS settings are for $2.5 \times 10^{10}$ particles, where the first 48 klystrons have a phase of -20 degrees and the remaining klystrons have a phase of +15 degrees. In all cases the overall linac phase was adjusted to make the energy spectrum correct at the end of the accelerator. At low current the oscillation decoheres because of the large energy spread. At $2.5 \times 10^{10}$ particles BNS damping is nearly correct because the oscillation damps approximately was the square root of the energy, as expected. At the highest current the BNS conditions are no longer effective and the oscillation starts to grow strongly at the end of the linac, similar to the examples in Figure 2. Thus, the BNS conditions must be matched to the beam current and lattice to make the technique most successful.

A beam oscillation can be used to determine lattice and beam parameters. By recording a beam trajectory and fitting the oscillation using Equation (2), an effective lattice strength can be 
determined ${ }^{10}$. A plot of the measured change in the effective lattice strength with current for the SLC is displayed in Figure 4. In an accelerator with 27 betatron wavelengths, a strength change of a few percent is quite noticeable.

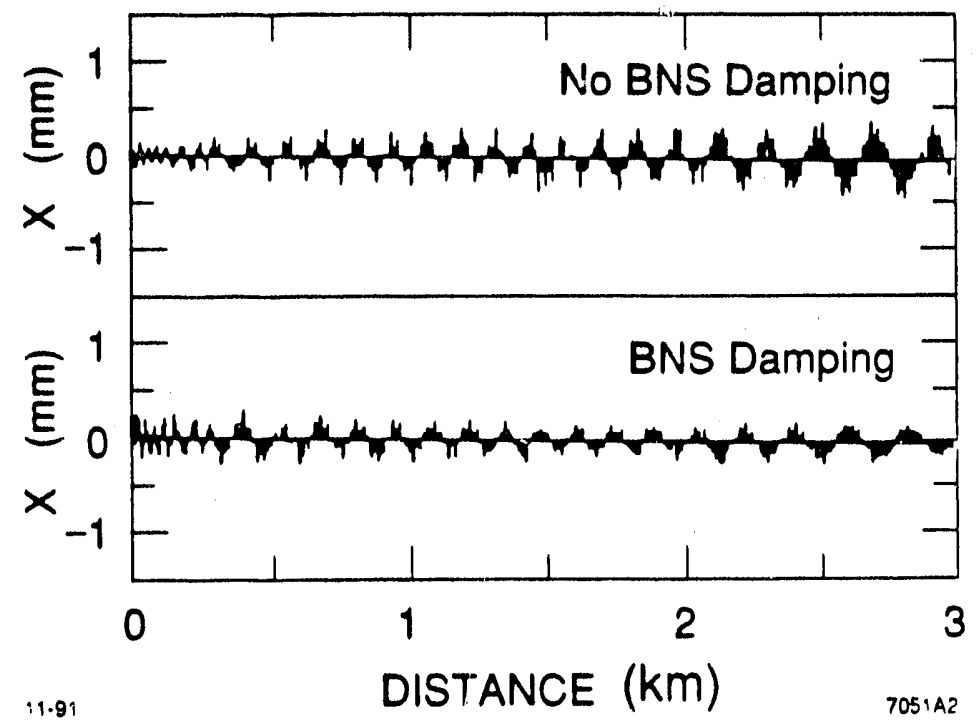

Figure 2 Measured oscillations in the SLC linac with and without transverse wakefield damping (BNS). The bunch charges are $2 \times 10^{10} \mathrm{e}^{-}$. The upper plot shows the exponential growth of the oscillation withrut BNS damping and the lower plot shows little growth with BNS damping. JII this example, the improvement in the ratio from the front to the end of the lin/c is a factor of ten.

\subsection{Autophasing}

The cancellation of wakefield forces by BNS damping may be exploited further, if a concept called autophasing can be made practical ${ }^{11}$. By careful arrangement of the bunch charge density based on knowledge of the local beam energy, lattice, bunch length, and RF structure, nearly all particles in the bunch can be made to follow exactly the same trajectory. The conditions for this behavior can be derived by substituting an identical oscillation, $x=x_{0} \sin \left(k_{0} s\right)$, into Equation (2) for all particles, and cancelling position terms on both sides ${ }^{2}$. The resulting autophasing condition is

$$
k^{2}(z, s)=k_{0}^{2}+\frac{r_{e}}{\gamma(z, s)} \int_{z}^{\infty} d z^{\prime} \rho\left(z^{\prime}\right) W_{r}\left(z^{\prime}-z\right)
$$

where $k_{0}$ is the desired effective lattice strength and $k(z, s)$ is the lattice strength for all particles if transverse wakefield forces are removed. With wakefields and the above condition, all particles have the same betatron frequency. The difficulty is that this condition must hold for all particles in the bunch, even the ones far forward and backwards off the RF crest, and at all locations along the accelerator. The adjustable parameters available are the bunch current, the lattice strength, the klystron energy profile, the klystron phases, and the longitudinal density profile. Of all these variables, the longitudinal density profile usually has the least number of constraints. However, in the SLC the early region has several lattice discontinuities which require beam energy spread changes which are more rapid than can be practically made. The irregularities of the lattice, the strong curvature of the RF voltage, and the need for a small energy spread at the end of the linac have made practical autophasing not fruitful to date ${ }^{2}$. although studies continue.

\subsection{Injection jitter tolerance}

The tolerance on injection parameters can be calculated with several goals in mind. One goal is to have the emittance of the beam not increase nacceptably during acceleration. (1) $\Delta \gamma \varepsilon / \gamma \varepsilon<0.1$. 


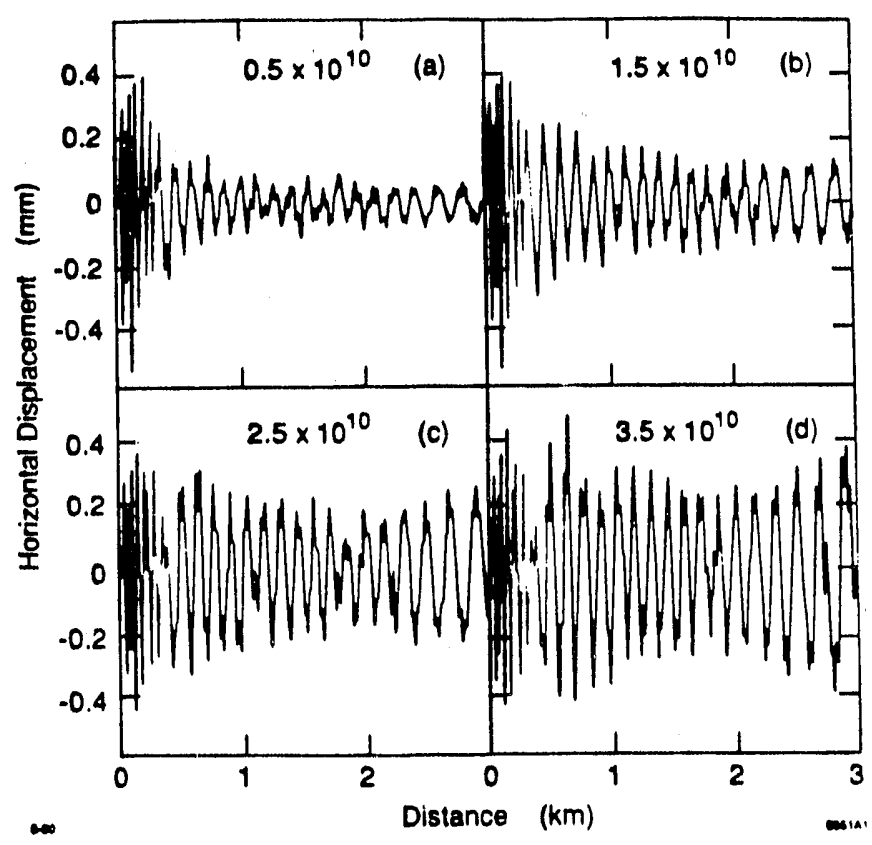

Figure 3

Observed single bunch oscillations along the SLC linac versus charge for identical dipole changes. Standard BNS conditions for $3 \times 10^{10} \mathrm{e}^{-}$are used in all cases with the overall linac phase adjusted to make a small energy spectrum at $47 \mathrm{GeV}$. Note that at high charge, damping from acceleration is not as strong as wakefield growth in the latter half of the linac.

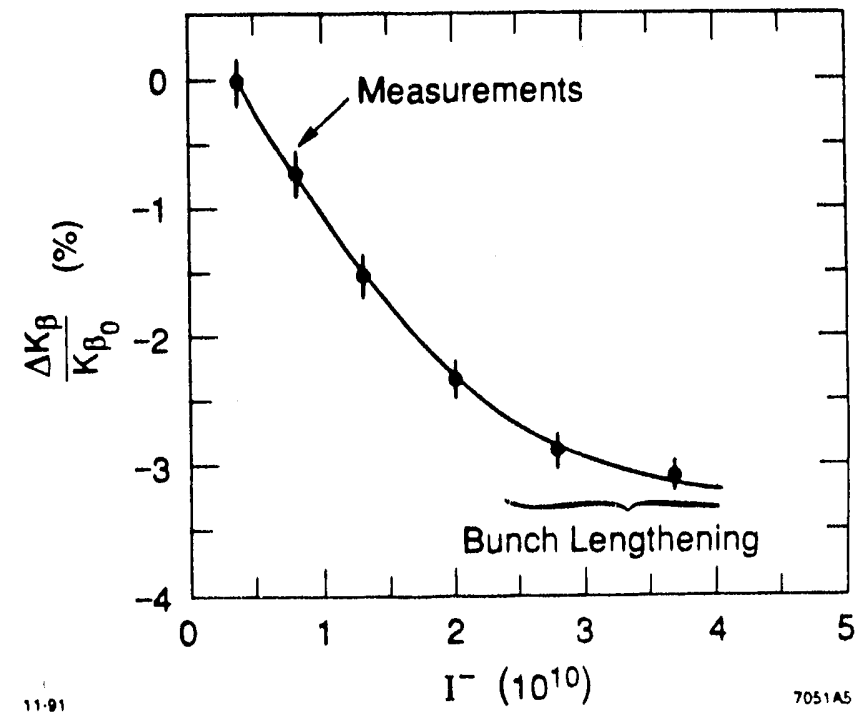

Figure 4 Observed apparent lattice strength changes as a function of beam current.

Another goal might be to keep the transverse position of the beam at the end of the accelerator within some fraction of the beam size from the accelerator axis to maintain luminosity. (2) $\Delta x<\sigma_{x} / 3$.

Conversely, (3) the goal may be to limit the number of particles a certain distance from the axis, as if to protect a device from destruction (i.e. collimator). A combination of these tolerances is used in the SLC in practice. A tolerance on injection position (and angle) jitter can be determined for the SLC from the data in Figure 3, with proper BNS conditions at each current using condition (2). The results ${ }^{12}$ of these calculations are shown in Figure 5. At $7.5 \times 10^{10}$ particles, the displacement at the beginning of the linac must be less that $40 \mu \mathrm{m}$, within a factor of two of present horizontal operational 


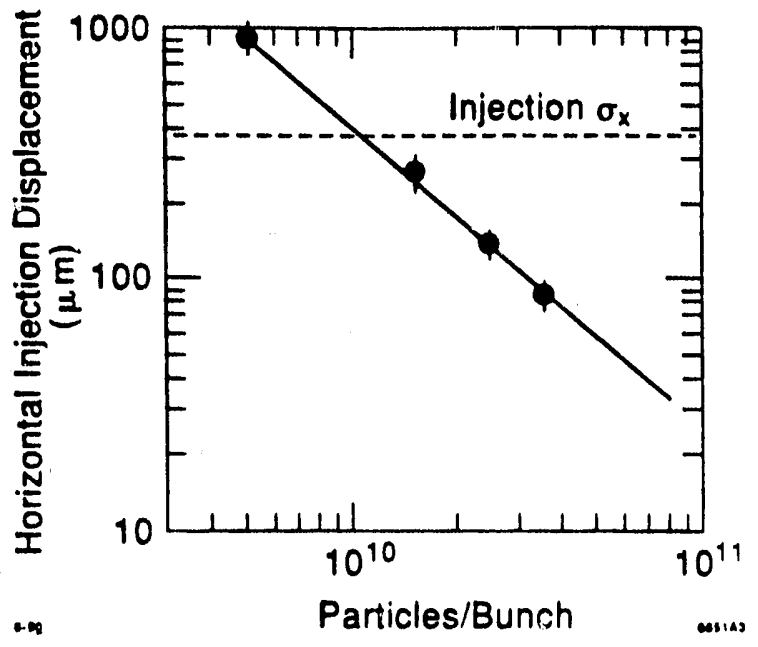

Figure 5 Injection tolerances versus bunch population as determined for the SLC using the data in Figure 3 and the requirement that the oscillation be smaller than one third the final bunch size.

values at $3 \times 10^{10}$. Vertically, the present injection jitter is satisfactory for all current values.

Feedback systems that work pulse-by-pulse are essential for keeping the beam parameters within acceptable limits given the many possible sources of transverse jitter. Several potential sources are varying power supplies of dipoles and off-axis quadrupoles, vibrating quadrupoles, klystron phase and amplitude jitter, dark current in the RF structure, unstable kicker magnets, temperature changes in the tunnel ${ }^{13}$, and changes in the beam intensity. In the SLC, over 80 beam parameters (beam positions, angles, and energies) are controlled by feedback routinely with many corrections each second. Not all parameters need rapid feedback. For example, the energy spectrum feedback has proven not to need pulse-by-pulse control. Modern control theory is used to provide cascaded control of position and angle loops from the beginning to the end of the accelerator that minimally interfere with each other and provide maximum control ${ }^{14}$. Reduction of oscillations with frequencies up to one sixth of the accelerator pulse rate can be expected.

Injection jitter too rapid for feedback control must be isolated and fixed. The sources of jitter are generally not stable. Thus, sophisticated analysis techniques must be developed. In the SLC the magnitude of the jitter can depend strongly on current. For example, the horizontal jitter becomes rapidly larger above $3.5 \times 10^{10} \mathrm{e}^{-}$per pulse. The cause for this rapid change is probably the large dispersion in the transport line between the damping ring and the linac combined with the lengthened bunch in the damping ring at high currents. The large amplitudes of the head and tail particles in the beam are not focused or bunched correctly. These particles then enter the linac far off-axis and far ahead or behind the main core of the beam. The head particles then excite wakefields downstream ${ }^{15,16}$. Correction of the second-order optical errors in the transport line is under way.

Dispersion at the entrance to the accelerator can cause the beam to have a transverselongitudinal tilt. $\Delta x=\eta \Delta E / E$. The head of the bunch is not on axis even though the average position of the bunch is. The oscillation of the head downstream will drive the trailing portions of the bunch to large amplitudes, increasing the emittance. Calculations of this effect for the SLC linac give a tolerance on the allowed dispersion as a function of current ${ }^{17}$. With BNS damping and charges of $3 \times 10^{10}$, the dispersion is controlled in the SLC to a few millimeters.

\subsection{Transverse profile and emittance changes with transverse wakefields}

Studies of beam transverse enlargement at the end of the SLC linac from betatron oscillations generated by a dipole magnet clearly show that leading particles which are off-axis in the accelerator excite trailing particles to larger amplitudes producing asymmetrical, skewed distributions. The particle distributions resulting from wakefields are not gaussian and, thus, must be described in a new way. Multiple gaussian fits, skewness, kurtosis, and fitting left. and right halves separately have been tried with various degrees of success. Also, an intuitive accelerator physics model for tail propagation can be 
described with an exponential tail which oscillates along the accelerator ${ }^{18}$. For a test, an oscillation was induced in an SLC electron beam with a charge of about $2.5 \times 10^{10} \mathrm{e}^{-}$, producing the trajectory shown in Figure 6. The associated beam profiles on four wire scanners downstream are shown in Figure 7. The observed beam shapes have a definite tail with a particular phase. The size and orientation of the transverse tail allow feedback control.

The beam, after the input parameters are optimized and the trajectory nominally corrected ${ }^{19}$, experiences emittance growth during acceleration because of alignment errors of the accelerator components. This results from the trajectory being steered through misaligned quadrupoles and accelerator structures onto beam position monitors with finite residual uffset errors. Methods to reduce these effects have been theoretically studied 20,21 . It was shown that the addition of appropriate injection errors $\left(\Delta x, \Delta x^{\prime}, \Delta y\right.$, and $\left.\Delta y^{\prime}\right)$ can cancel most of the emittance enlargement. Since the advent of BNS damping, a more global scheme of distributing short range oscillations along the accelerator has been shown to be satisfactory 22 . Oscillations have been successfully added to the SLC linac to improve colliding beam operations ${ }^{19}$.

At high currents the alignment of the RF structure relative to the quadrupoles and position monitors dominates all other alignment issues. Great efforts have been made to develop techniques to

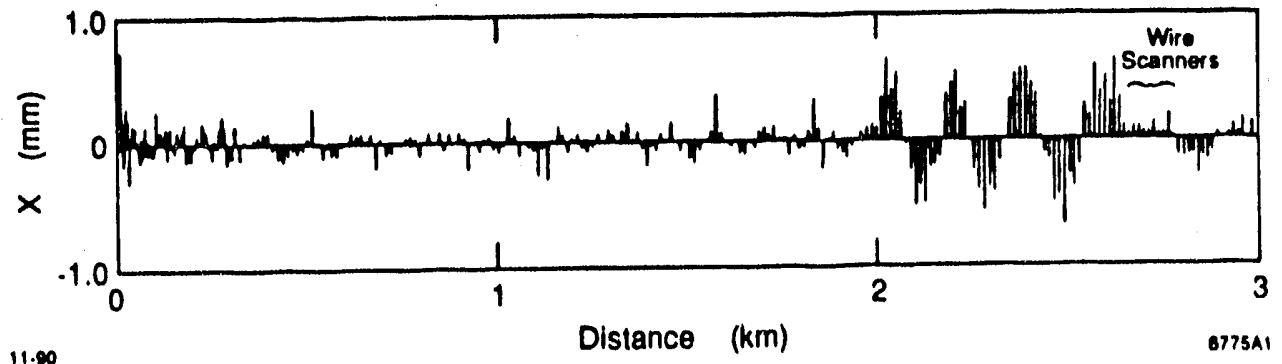

Figure 6 A particular beam oscillation used to generate the transverse beam tails in Figure 7.

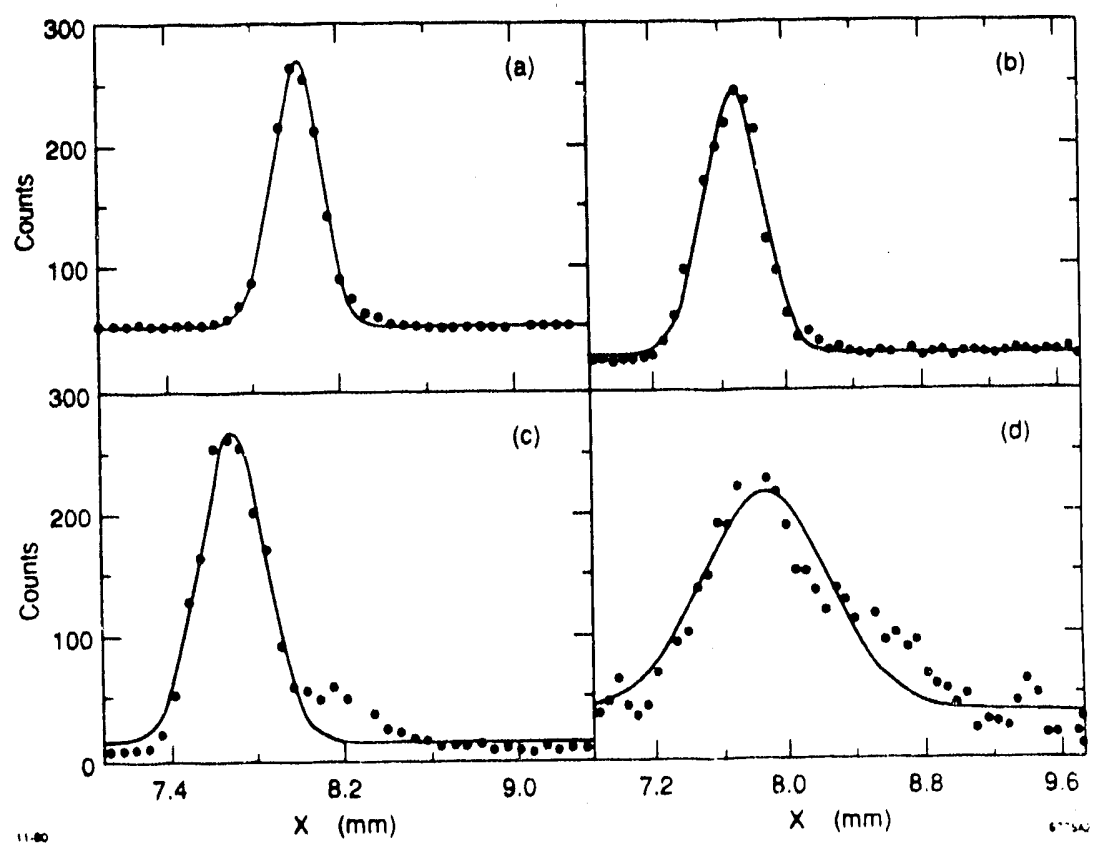

Figure 7 Measured beam profiles ( $47 \mathrm{GeV}$ ) in the SLC for the betatron oscillation in Figure 6. The projections were taken with four wire scanners at (a) 0 , (b) 22.5 , (c) 90 ., and (d) 112.5 degrees in betatron phase. Note that the induced transverse tail has a particular phase such that the tail does not appear at position (a). 
align the RF structure to $100 \mu \mathrm{m}$ or less over many kilometers of linac and to keep them aligned over a long time period with minimal effort ${ }^{23}$. Experimentally, it has been shown that moving structure irises can deflect a $6 \mathrm{GeV}$ beam ${ }^{24}$, as is shown in Figure 8. These observations have naturally directed efforts towards a possible control. The component of the structure alignment errors at the betatron spatial frequency is primarily that which drives the beam. Therefore, if a control mechanism can be made to move the structure at that spatial frequency, then sine and cosine adjustments can be used to cancel the accumulation of alignment errors of the accelerator ${ }^{25}$. The initial alignment tolerances can then be relaxed. This control can also be used to correct for any long term position drifts of the accelerator supports due to temperature changes or floor creep in the tunnel. A $100 \mathrm{~m}$ test of this active control is underway in the SLC Linac.

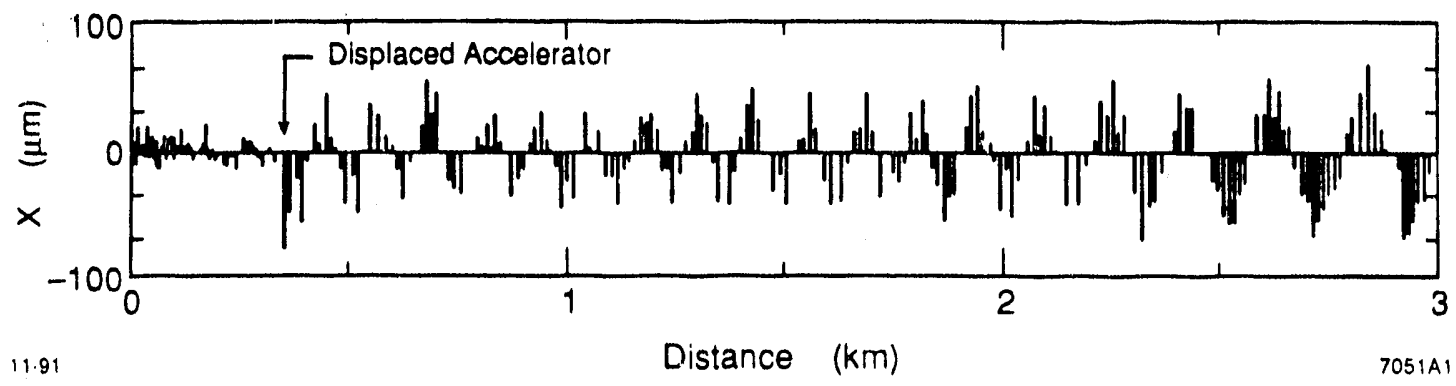

Figure 8 The center of an RF accelerating girder (12 $\mathrm{m}$ ) of the SLAC linac was displaced by $1 \mathrm{~mm}$ while leaving all other parameters fixed. The observed transverse oscillation of a beam downstream resulted from the displacement of copper irises only.

\subsection{Acknowledgments}

The SLC Linac and Emittance Study Groups provided many stimulating discussions.

\subsection{References}

1. J. Seeman, et al., SLAC-PUB-5437, Stanford (1991).

2. J. Seeman and L. Merminga, 1990 Linear Accelerator Conference, Albuquerque, p. 387 (1990).

3. K. Bane and P. Wilson, 11th Int. Conf, on High Energy Accelerators, Birkhauser, p. 592 (1980).

4. J. Seeman, et al., SLAC-PUB-5438, Stanford (1991).

5. Z. D. Farkas, et al, 9th Int. Conf, on High Energy Accel., p. 576 (1974).

6. K. Bane, SLAC/AP-80, Stanford (1990).

7. J. Seeman, et al., IEEE CH2387-9/87/0000, Washington PAC, p. 73 (1987).

8. V. Balakin, et al., 12th Int. Conf. on High Energy Accel., FNAL, p. 119 (1983).

9. J. Seeman, et al., SLAC-PUB-4968, Stanford (to be published).

10. C. Adolphsen, et al., SLAC-PUB-5581, Stanford (1991).

11. V. Balakin, 1988 Workshop on Linear Colliders, SLAC, p. 119 (1988).

12. T. Limberg, et al., EPAC, Nice, p. 1506 (1990).

13. J. Seeman, et al., SLAC-PUB-5439, Stanford (1991).

14. T. Himel, et al., SLAC-PUB-5470, Stanford (1991).

15. M. Ross, private communication.

16. J. Seeman, et al., SLC-Reports-249 and 252, Stanford (1991).

17. J. Seeman, SLAC-CN-330, Stanford (1986).

18. J. Seeman, SLAC-PUB-5440, Stanford (1991).

19. J. Seeman, "SLC Beam Dynamics Issues", 5th ICFA Workshop, Corpus Christi (1991).

20. A. Chao, B. Richter, and C. Yao, Nucl. Inst. and Meth. 178, p. 1 (1980).

21. K. Bane, IEEE Trans. Nucl. Sci. NS-32, No. 5, p. 2389 (1985).

22. J. Seeman, et al., SLAC-PUB-5705, Stanford (1991)

23. J. Seeman, et al., SLAC-PUB-5439, Stanford (1991).

24. J. Seeman, SLAC-PUB-5706, Stanford (1991)

25. J. Seeman, 1990 Linear Accelerator Conference, Albuquerque, p. 387 (1990). 

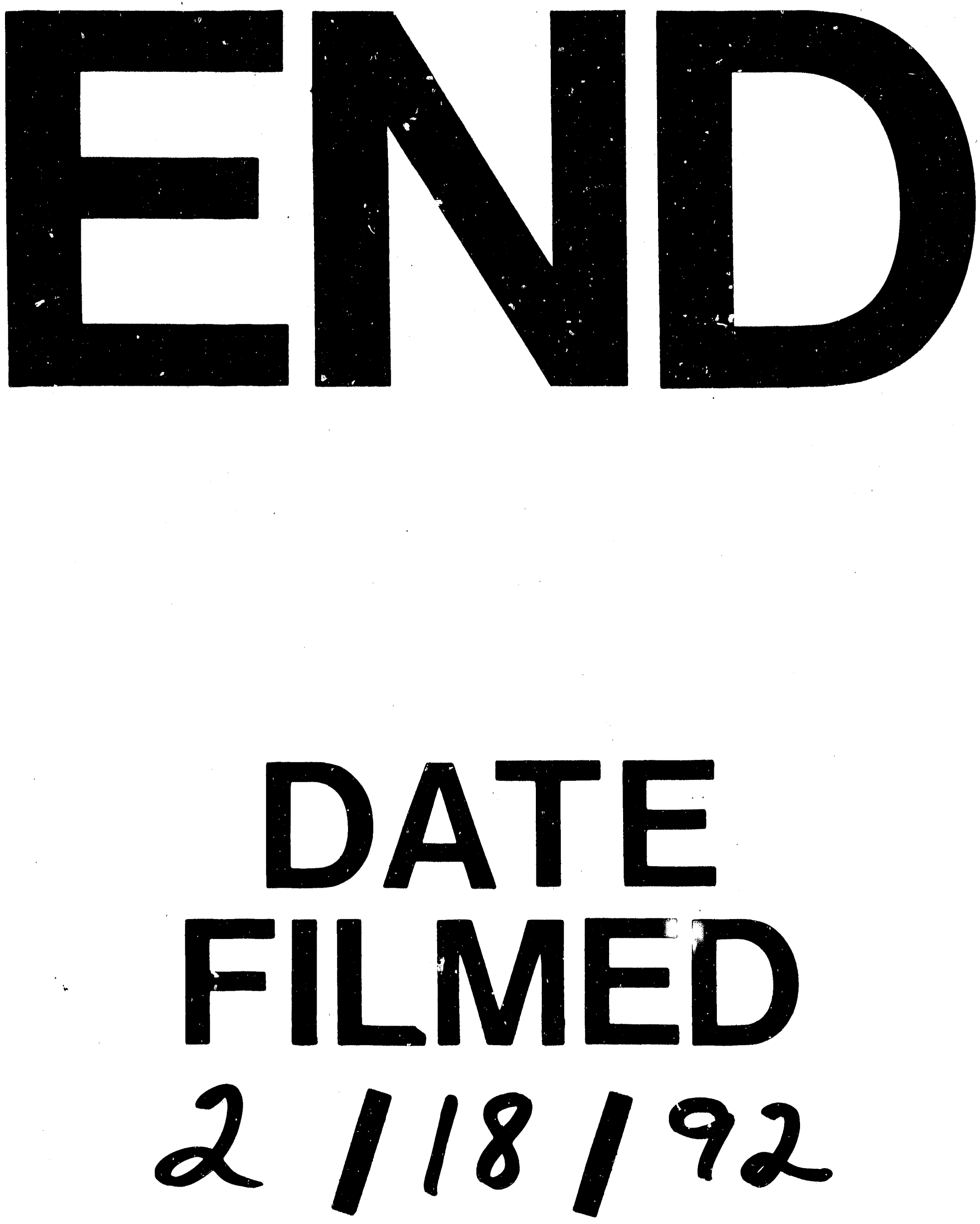
. 